\title{
ESTUDIO Y EVALUACIÓN DE LOS MODELOS MATEMÁTICOS PARA LA ESTIMACIÓN DE LA DILATANCIA
}

\author{
Victor Hugo Rondón ${ }^{1 *}$, Miguel Ángel Fuentes², Yair Andrés Quintero ${ }^{3}$ \\ *A quien debe dirigirse la correspondencia
}

\section{RESUMEN}

El artículo muestra una revisión bibliográfica de los principales modelos que existen para el cálculo de la dilatancia en la roca. Una vez explicado cada modelo, se procede a realizar el modelamiento matemático de ésta propiedad. Esto se realiza mediante datos suministrados por la literatura, las cuales son: esfuerzos sometidos a la roca $\sigma 1, \sigma 2, \sigma 3$, ángulo de fricción interna $\emptyset$, pendiente de la línea de estado critico $\mathrm{M}$, el parámetro de estado , y otras constantes que se requieren para cada modelo. Una vez hechos los cálculos de la dilatancia en la roca, se procede a validar el modelamiento con datos reales, los cuales también son suministrados por la literatura. En éstas pruebas se midió la dilatancia real en dos tipos de arena, (Banding y Miga) y se realizaron 5 pruebas. Finalmente éstas mediciones se compararon con los cálculos realizados, y así llegar a la conclusión de que el mejor modelo para predecir el fenómeno dilatante de una roca es el de Li \& Dafalias.

Palabras clave: Arenamiento, dilatancia, estado crítico, suelos granulares.

\section{STUDY AND EVALUATION OF MATHEMATICAL MODELS FOR ESTIMATING DILATANCY}

\begin{abstract}
The article shows a literature review of the main models that exist for the calculation of dilatancy in the rock, which is a property that has an application in the study of the phenomenon of sanding in oil wells. Having explained each model, we proceed to perform mathematical modeling of this property. This is done using data supplied by the literature, which are: strain in the rock $\sigma 1, \sigma 2, \sigma 3$, internal friction angle $\emptyset$, slope of the critical state line $M$, the state parameter $\varphi$, and other constants which are required for each model. Once dilatancy calculations are made in the rock, we proceed to validate the modeling with real data, which are also supplied by the literature. In these tests, the dilatancy was measured in two types of sand, (Banding and crumb) from which 5 tests were done; these measurements were compared with calculations, and finally concludes that the best model to predict the dilatant rock phenomenon is Li \& Dafalias.
\end{abstract}

Keywords: Sanding, dilatancy, critical condition, granular soil.

1. Universidad Industrial de Santander, UIS. Bucaramanga, Colombia. E-mail: victorhugorondon@gmail.com

2. Universidad Industrial de Santander, UIS. Bucaramanga, Colombia. E-mail: ingmafb@gmail.com

3. Instituto Colombiano del Petróleo, ICP. Bucaramanga, Colombia. E-mail: yair.quintero@ecopetrol.com.co 


\section{INTRODUCCIÓN}

El arenamiento es uno de los principales problemas que existen en un pozo, donde las arenas son poco consolidadas o donde los caudales de gas producidos son muy grandes ocasionando inestabilidad del mismo, colapso de la tubería de revestimiento, una reducción en la producción y en casos extremos la pérdida del pozo [44], razón por la cual es de suma importancia tratar de comprender éste fenómeno, y a su vez saber cómo se genera y cómo se podría controlar. [33]

Cuando se produce arena, ésta por lo general está asociada a tres procesos principalmente, falla por cizallamiento de la roca, falla por tensión de la roca y/o fuerzas de arrastre del fluido de producción-inyección [20], siendo para nuestro interés las condiciones de cizallamiento, la cual está afectada principalmente por los esfuerzos de la superficie, esfuerzos generados durante la perforación, esfuerzos inducidos durante el completamiento y por la producción como tal; además de la relación con la presión de poro y la resistencia de la roca [7]. En este momento es donde entra el concepto de dilatancia, debido a que está ligado directamente con la falla en la roca: a mayor dilatancia, menor nivel de compactación en la roca, y por ende menor resistencia [4]. Al tener menor resistencia la roca, ésta fallará más fácil mente, esto puede ocurrir mientras se está perforando o mientras se está produciendo crudo.

En Colombia no se ha realizado un estudio sobre el fenómeno de dilatancia, el cual es de suma importancia, ya que éste concepto está ligado con el criterio de falla, y por consiguiente con la estabilidad del pozo, ya sea mientras se perfora o mientras se produce crudo.

\section{DILATANCIA}

Cuando una roca densa es sometida a esfuerzos sus partículas internas empiezan a tener un cambio de posición, pasan de ser partículas muy unidas o compactadas, a partículas separadas [5]; ésta separación hace que la roca en general tenga un aumento de volumen, a este proceso se le llama dilatancia [34], sin embargo, cuando la arena es suelta sucede el proceso contrario, al reacomodarse las partículas lo que hacen es unirse y los espacios que hay entre cada grano se disminuyen, de ésta forma el volumen de la roca en general también lo hace, en este caso se presentaría una dilatancia negativa o contracción [21]. Este reordenamiento interno ocurre porque una partícula se desliza encima de la otra, para a veces quedar más separadas o más unidas dependiendo de la condición inicial de la roca. [29] Al aumentarse el volumen y reordenarse las partículas se crean espacios entre cada grano, el cual se puede llenar de agua, aceite o cualquier tipo de fluido, este se filtra hacia el espacio que se ha generado [47]

Cuando ocurre la dilatancia, la permeabilidad y la porosidad se ven afectadas ya que se inducen cambios en los espacios entre los granos [49], esto hace que la resistencia de la roca disminuya y la roca falle más rápido [11]. Existe una configuración inicial de los granos sometidos a esfuerzos, donde hay un área critica entre cada grano, ésta va cambiando su dirección a medida que los esfuerzos cambian, esto hace que se modifique la permeabilidad [3].

\section{APLICACIONES}

Como se explicó, el fenómeno de dilatancia tiene influencia en el cambio de permeabilidad y porosidad en una roca, este cambio puede ser de gran utilidad para producir crudo con mayor eficiencia [27]. Por ejemplo, el método SAGD, el cual es un proceso donde dos pozos horizontales paralelos son perforados en la formación, y uno de ellos se encuentra 5 metros por encima del otro [28]. Donde al pozo superior se le inyecta vapor y en el pozo de abajo se acumula el petróleo caliente que se drena desde la formación productiva, junto con el agua de la condensación del vapor inyectado [38]. La base del proceso es que al inyectar el vapor se forma una cámara de vapor que crece a lo alto de la vertical y horizontalmente en la formación. El calor del vapor reduce la viscosidad del crudo pesado, lo cual permite que fluya hacia la parte inferior del pozo [43].

Geomecánicamente, cuando éste proceso ocurre, el aumento de la temperatura hace que las rocas se dilaten [37], éstas a su vez generan esfuerzos sobre las otras rocas que están alrededor, se reordenan, y se presenta el fenómeno de dilatancia mecánica [8]. Este reordenamiento hará que la permeabilidad aumente, gracias a esto, habrá mayor facilidad para que el crudo pesado fluya [10].

\section{CRITERIO DE FALLA - DILATANCIA}

Existe una relación entre el criterio de falla de la arena y la dilatancia de ésta misma. La tendencia a que la roca falle es mucho más alta cuando la dilatancia es elevada, ya que las partículas internas están más separadas y esto hace que surja un comportamiento más blando en la arena. [16] Para establecer las condiciones en que se 
establece la falla, se utiliza el modelo de Mohr Coulomb [18] a fin de relacionar los esfuerzos principales y la presión de poro con la cohesión y el ángulo de fricción interna. En una muestra de roca a la cual se le hace ésta prueba, va a mostrar un comportamiento especifico dependiendo de los esfuerzos aplicados [19], este comportamiento es modificado cuando la dilatancia está presente, ya que la muestra se tornará más blanda, (separación interna de partículas) y esto hará que la roca se falle mucho más rápido, lo cual provocará que haya un cambio entre la línea de falla y el circulo de Mohr [23].

\section{DENSIDAD-DILATANCIA}

La densidad en la roca es una propiedad indispensable para la medida de la dilatancia, ya que dependiendo de ésta se podrá saber cómo se comportará la arena, por ejemplo, si es una arena muy densa la arena tenderá a dilatarse, mientras que si es una arena suelta ésta tenderá a contraerse [31].

\section{MODELOS PARA EL CÁLCULO DE LA DILATANCIA}

\section{MODELO DE TAYLOR}

La primera persona que empezó con los estudios de dilatancia fue Taylor en 1948 [45], donde definió y explico el concepto de dilatancia mediante el tradicional ensayo de corte, él sugirió que la dilatancia dependía de los esfuerzos y de una constante friccional típica de cada material. Su cálculo lo pudo representar en la ecuación 1:

$$
d=\mu-n
$$

Donde:

$d=$ Dilatancia. [Adimensional]

$\mu=$ Constante friccional. [Adimensional]

$n=$ Relación de tensiones. [Adimensional]

Este modelo simple fue la primera expresión de dilatancia que se trabajó, para Taylor el único efecto directo sobre el aumento de volumen son los esfuerzos [24].

\section{MODELO CAM CLAY (SCHOFIELD \& WROTH (1963))}

Wroth y Schofield en 1963 [48] emplean una segunda expresión para el cálculo de dilatancia la cual se representa en la ecuación 2:

$$
d=M-n
$$

Donde:

$M=$ Pendiente de línea del estado critico [Adimensional]

$n=$ Relación de tensiones. [Adimensional]

$d=$ Dilatancia [Adimensional]

Cada parámetro se obtiene de la siguiente manera:

\section{Tensión desviadora "q":}

Es una relación de esfuerzos y se puede calcular con la ecuación 2.1:

$$
q=\sigma 1-\sigma 3
$$

Donde:

$\mathrm{q}=$ Tensión desviadora $[\mathrm{KPa}]$

$\sigma 1=$ Presión normal o Axial $[\mathrm{KPa}]$

$\sigma 3=$ Presión lateral aplicada [KPa] [6]

Tensión volumétrica efectiva p':

Se calcula con la ecuación 2.2:

$$
p^{\prime}=\frac{\sigma 1+\sigma 2+\sigma 3}{3}
$$

$p{ }^{`}=$ Tensión volumétrica $[\mathrm{KPa}]$

$\sigma 1=$ Presión normal o Axial $[\mathrm{KPa}]$

$\sigma 3=$ Presión lateral aplicada $[\mathrm{KPa}]$

$\sigma 2=$ Presión lateral aplicada $[\mathrm{KPa}]$

En este caso (ecuación 2.2), p' tiene en cuenta los 3 esfuerzos a los que es sometida la roca y se divide en 3 (promedio).

\section{Relación de tensiones $n$}

Teniendo estos dos parámetros se puede llegar a calcular la relación de tensiones $n$ mediante la ecuación 3 [13]. 


$$
n=\frac{q}{p}
$$

Donde

$\mathrm{n}=$ Relación de tensiones [Adimensional]

$\mathrm{q}=$ Tensión desviadora $[\mathrm{KPa}]$

$\mathrm{p}^{\prime}=$ Tensión volumétrica efectiva $[\mathrm{KPa}]$

\section{Pendiente de línea del estado crítico $M$.}

Cuando se grafica la tensión desviadora contra la tensión volumétrica efectiva y se va variando un esfuerzo se crea una línea recta, a ésta se le llama línea del estado crítico [41].

Para calcular la pendiente de ésta línea hay dos formas, la primera, para diferentes presiones de confinamiento o ensayos triaxiales que es sometida la roca se obtiene la presión a la cual la roca pasa de presentar un comportamiento elástico a plástico, se toma los puntos de $\mathrm{p}$ y q a ésta presión, se hace lo mismo para las diferentes pruebas triaxiales y finalmente se traza una línea que pasa desde cero (0) interceptando esos puntos. La pendiente se obtiene como cualquier línea recta. [42]

Otra forma es utilizando la ecuación 4:

$$
\sin \varnothing=\frac{3 M}{6+M}
$$

Donde:

$\varnothing=$ Ángulo de fricción interna [Grados]

$\mathrm{M}=$ Es la pendiente de la línea del estado crítico [Adimensional] [1].

Estos son todos los parámetros que se necesitan para poder utilizar el modelo Cam Clay (ecuación 2) además de la relación de tensiones también empleada en el modelo de Taylor (ecuación 1). Las demás ecuaciones para calcular la dilatancia también los emplean con algunas modificaciones o agregando otros parámetros que serán explicados en su momento [2].

\section{MODELO CAM CLAY MODIFICADO ROSCOE \& BURLAND (1968)}

Después de varios años de estudio del modelo Cam Clay fue modificado para llegar a cálculos más precisos [14], esto debido a que la ecuación predice deformaciones plásticas desviadoras importantes solo para pequeños valores de $\mathrm{h}$ (magnitud escalar denominada modulo plástico) [15] por lo que se sustituyó por la ecuación 5.

$$
d=\left(M^{2}-n^{2}\right) / 2 n
$$

Donde

$\mathrm{M}=$ Pendiente de la línea del estado crítico. [Adimensional]

$n=$ es la relación de tensiones [Adimensional] [9].

Por varios ensayos triaxiales Roscoe y Burland en 1968 [39] pudieron llegar a la ecuación 5 para el cálculo de la dilatancia, la cual era mucho más exacta que la planteada en la ecuación 2. Este nuevo modelo no varía con respecto a los parámetros utilizados para los cálculos, solo se tiene una nueva restructuración de la formula, donde según Roscoe y Burland [39] predicen mejor el comportamiento dilatante que el modelo Cam Clay original (ecuación 2).

\section{MODELO DE ROWE'S}

En 1962 Rowe [40] determinó otra ecuación para el cálculo de dilatancia basada en la idea de que la relación del trabajo realizado por una fuerza que produce un incremento de deformación en el suelo y el trabajo disipado al producirse este debe tomar un valor de constante $\mathrm{K}$, relacionado directamente con el ángulo de fricción interna $\Phi$.

El valor de este ángulo varía entre un mínimo correspondiente al ángulo de rozamiento movilizado cuando solo algunas partículas se están deslizando sobre otras, y un máximo relacionado con el ángulo de resistencia al corte en el estado crítico, cuando se están produciendo grandes deformaciones en el suelo y su estructura se está reordenando constantemente [31].

La relación que Rowe plantea (ecuación 6), donde " $k$ " está en base al ángulo de fricción interna, es la siguiente:

$$
k=\tan ^{2}\left(\frac{\pi}{4}+\frac{\varnothing}{2}\right)=\frac{1+\sin \varnothing}{1-\operatorname{sen} \varnothing}
$$

Donde:

$k=$ Parámetro en función del ángulo de fricción interna [Adimensional].

$\varnothing=$ Ángulo de fricción interna [Grados]

En la ecuación 6 se puede observar que este parámetro $k$ está relacionado con el ángulo de fricción interna, el cual está denotado por ø, ésta es la primera consideración que hace el autor del modelo para llegar finalmente a la ecuación que describe la dilatancia [46]. 
Una segunda consideración es la definición de dilatancia presentada en la ecuación 7

$$
d=\frac{d \varepsilon v}{d \varepsilon s}
$$

Donde:

$\mathrm{d} \varepsilon \mathrm{v} / \mathrm{d} \varepsilon s=$ Razón de incremento desviadores $\mathrm{y}$ volumétricos de deformación plástica. [Adimensional]. $d=$ dilatancia [Adimensional]

Rowe [40] llega a la ecuación final que representa el fenómeno de dilatancia en la roca (ecuación 8), esto se hace teniendo en cuenta las dos anteriores consideraciones (ecuación 6 y 7), desarrollando un largo proceso matemático que en este artículo no es el objetivo demostrar:

$$
d=\frac{d \varepsilon v}{d \varepsilon s}=\frac{9(M-n)}{9+3 M-2 n M}
$$

Donde:

$M=$ Pendiente de línea del estado critico [Adimensional] $n=$ Relación de tensiones. [Adimensional]

$d=$ Dilatancia [Adimensional]

\section{MODELO DE NOVA}

Éste modelo matemático se publica en el año 1979 propuesto por Nova y Wood [35]. Donde teniendo en cuenta la relación de tensiones " $n$ ", proponen calcular la dilatancia como se muestra en la ecuación 9:

$$
d=\frac{(M-n)}{(1-N)}
$$

Donde:

$M=$ Pendiente de línea del estado critico [Adimensional]

$n=$ Relación de tensiones. [Adimensional]

$d=$ Dilatancia [Adimensional]

$\mathrm{N}=$ Coeficiente de acoplamiento volumétrico [Adimensional]

En la ecuación 9 se puede observar que solo depende de los esfuerzos y un parámetro $\mathrm{N}$ integrado, al cual se le llamó coeficiente de acoplamiento volumétrico.

En el caso particular de que $\mathrm{N}=0$, el modelo quedaría exactamente igual al de Cam Clay (ecuación 2). N está en un rango entre 0 y 1 ; existe en la literatura un valor de $\mathrm{N}$ para cada tipo de arena obtenidos de calibración por pruebas de laboratorio, sin embargo se puede calcular con la ecuación 10 , teniendo la pendiente de línea de estado crítico y la constante Do que se obtiene por calibración cuando se supone que las deformaciones elásticas son pequeñas y despreciables:

$$
D o=M /(1-N)
$$

Donde:

$M=$ Pendiente de línea del estado critico [Adimensional] $\mathrm{N}=$ Coeficiente de acoplamiento volumétrico [Adimensional]

Do $=$ Parámetro de calibración [Adimensional $]$

Se define Do en la ecuación 10.1:

$$
D o=d o / \mathrm{M}
$$

Donde:

$M=$ Pendiente de línea del estado critico [Adimensional] Do $=$ Parámetro de calibración [Adimensional] $d o=$ Constante, generalmente 0.88

El parámetro "do" proviene del modelo constitutivo Li and Dafalias, y se obtiene por calibración de la prueba de laboratorio, en general tiene un valor de 0.88 , aunque cambia para el tipo de arena.

\section{MODELO DE GAJO Y WOOD}

En 1999 Gajo y Wood [17] llegan a otra ecuación para calcular el fenómeno de dilatancia, donde la línea de transformación de fase va variando con el parámetro de estado de forma lineal, la ecuación 11 muestra el modelo matemático a que se llegó:

$$
d=A *\left(M *\left(1+k_{d} * \varphi\right)-n\right)
$$

Donde:

$M=$ Pendiente de línea del estado critico [Adimensional]

$n=$ Relación de tensiones. [Adimensional]

$d=$ Dilatancia [Adimensional]

$\mathrm{A}=$ Factor multiplicador de la regla de flujo. [Adimensional]

$\Phi$ = Parámetro de estado.[Adimensional]

$\mathrm{Kd}=$ Parámetro de ajuste en el estado de la regla de flujo. [Adimensional] 
En este modelo constitutivo (ecuación 11), los esfuerzos, el ángulo de fricción interna y la porosidad están incluidos también con la relación de tensiones desviadoras y volumétricas, también la pendiente de línea de estado crítico “ $M$ ” y el parámetro de estado que es la relación de porosidades inicial y la porosidad en el punto de estado crítico.

Además hay dos parámetros más que se incluyen en este modelo matemático (ecuación 11), “ $A$ ” que es un multiplicador de la regla de flujo, y " $k d$ " que es otro parámetro de ajuste en el estado de la regla de flujo.

Donde $\mathrm{A}=1, \mathrm{y} \mathrm{Kd}=1.1$ aproximadamente para la mayoría de las arenas.

Pero También, para calcular Kd se puede utilizar la ecuación 11.1:

$$
k_{d}=\frac{1}{\varphi} *\left(\frac{n}{M}-1\right)
$$

Donde:

$M=$ Pendiente de línea del estado critico [Adimensional]

$n=$ Relación de tensiones. [Adimensional]

$\Phi=$ Parámetro de estado. [Adimensional]

$\mathrm{Kd}=$ Parámetro de ajuste en el estado de la regla de flujo. [Adimensional]

El parámetro de estado $\varphi$, está comprendido por la resta de la relación de vacíos como se observa en la ecuación 11.2 .

$$
\varphi=e-e_{c}
$$

Donde:

$e=$ Relación de vacíos inicial [Adimensional]

$e_{c}=$ Relación de vacíos critica [Adimensional]

Éstas son mediciones que se hacen en laboratorio, la primera se toma al inicio de la prueba y la segunda justamente cuando se toca la línea de estado crítico. Éstas medidas tienen que ver con la porosidad de la roca [50].

\section{MODELO DE LI Y DAFALIAS}

En este modelo (ecuación 12) se analiza que la dilatancia no puede depender únicamente de la relación de tensiones como se observó en los modelos explicados [32].
La expresión que calcula la dilatancia, está dada por la ecuación 12:

$$
d=d o *((\exp (m * \varphi))-n)
$$

Donde:

$n=$ Relación de tensiones. [Adimensional]

$d=$ Dilatancia [Adimensional].

$\varphi=$ Parámetro de estado. [Adimensional]

$d o=$ parámetro de calibración [Adimensional]

$m=$ Parámetro asociado a línea de transformación de fase [Adimensional]

A medida que se va avanzando, se puede ver que los modelos matemáticos van mostrando nuevos parámetros, en este caso $m$ y do que son los únicos diferentes. Estos dos varían dependiendo del tipo de arena. Al final en el anexo se mostraran las tablas con los diferentes parámetros para los diferentes tipos de arena. [22]

\section{MODELO DE PASTOR ET AL}

Planteado por Pastor en 1986 [36] plantea el cálculo de la dilatancia mediante la ecuación 13 :

$$
d=(1+\alpha) \cdot(M-n)
$$

$M=$ Pendiente de línea del estado critico [Adimensional] $n=$ Relación de tensiones. [Adimensional]

$d=$ Dilatancia [Adimensional]

$\alpha g=$ Constante, generalmente 0.45

Además de que la dilatancia sigue dependiendo de la configuración de esfuerzos sometidos a la roca, para este modelo (ecuación 13) se emplea una constante $\alpha$ g, que está dada para los diferentes tipos de arena, pero que en su mayoría es 0.45 .

\section{MODELAMIENTO}

Una vez se conocen los principales modelos matemáticos para el cálculo de la dilatancia se procede a probar cada uno para saber cuál es el que mejor representa el fenómeno. Para esto se tomaron datos experimentales de otros documentos disponibles en la literatura, con muestras y características de la prueba diferentes, con el fin de comparar los resultados obtenidos con cada modelo.

A continuación se muestra cada uno de los ensayos con sus características. 


\section{ARENA MIGA}

Ésta arena está constituida por arcosas miocenas y cuarzo feldespáticas.

Se escogieron datos de una tesis doctoral publicada en el 2008 en Madrid por Diego Guillermo Manzanal [12], Donde hay mediciones de dilatancia reales. Allí existen dos secciones de arena miga denominadas 4478 y 4479.

Para probar los modelos de dilatancia con ésta arena, se tomó la muestra 4479-E TCD50. Es una arena sometida a un ensayo triaxial drenado, con una presión de confinamiento de $50 \mathrm{Kpa}$.

Se hizo una gráfica de dilatancia (eje $\mathrm{x}$ ) vs la relación de tensiones $n$ (eje y), donde se graficaron las medidas de dilatancia sacadas del laboratorio y las calculadas por las ecuaciones.

En la Figura 1 se puede observar la dilatancia medida en laboratorio (rojo) y los modelos matemáticos que son los demás colores, el que mejor se acerca al comportamiento real es el de Li and Defalias (naranja), aunque los otros modelos no muestran una desviación muy grande con respecto al comportamiento real.

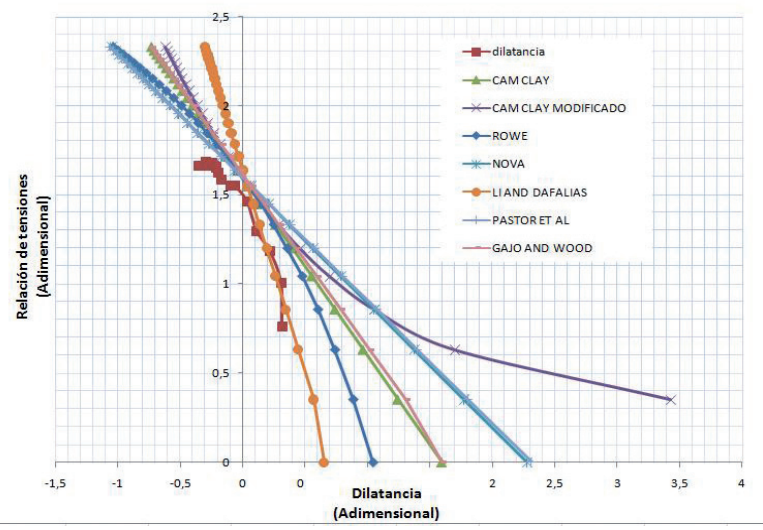

Figura 1. Modela miento del fenómeno de dilatancia muestra 4479-E TCD50

\section{ARENA MIGA 4479-A TCD 300}

Para ésta segunda medida de dilatancia se trabaja una muestra diferente pero del mismo tipo de arena "miga". Ahora con una presión de confinamiento de $300 \mathrm{Kpa}$, con el fin de ver el comportamiento de cada uno de los modelos a diferentes presiones. Ver Figura 2.

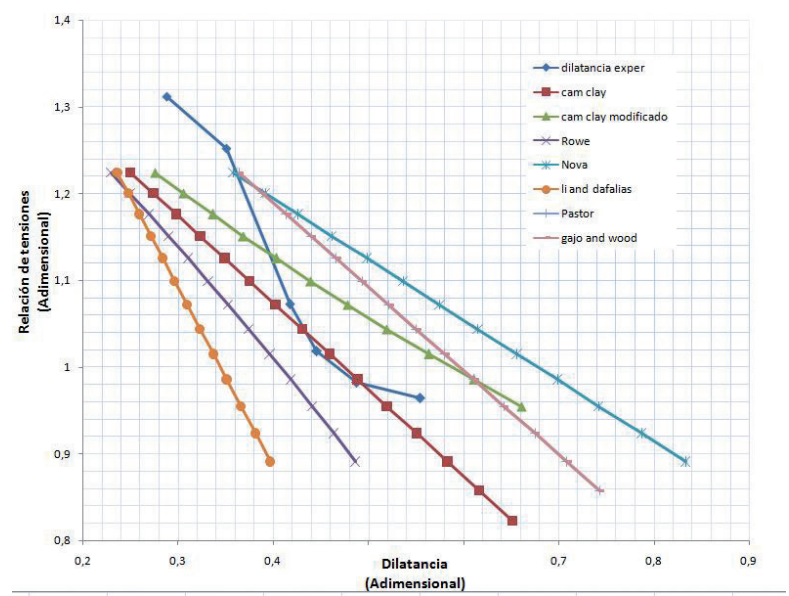

Figura 2. Modelamiento del fenómeno de dilatancia muestra 4479-A TCD 300

De nuevo la dilatancia mediada en laboratorio (azul oscuro) es presentada, pero esta vez los modelos no ajustan muy bien al comportamiento, a medida que se aumenta el esfuerzo en la simulación, las ecuaciones tienden a alejarse del comportamiento real en la roca.

\section{ARENA BANDING B 13 (CASTRO 1969)}

En el artículo de Been \& Jefferies [25] se analizaron dos tipos de arena: Erksak y la "sand Banding". La arena Banding de castro (1969) fue estudiada como tesis doctoral en Harvard. Castro fue el primero en evaluar la licuefacción en arenas. La arena B13 de castro es una arena suelta el cual muestra un comportamiento dilatante diferente, pero que trata de ajustarse a un particular modelo. Para este caso, se trabaja con una presión inicial de confinamiento de $60 \mathrm{Kpa}$.

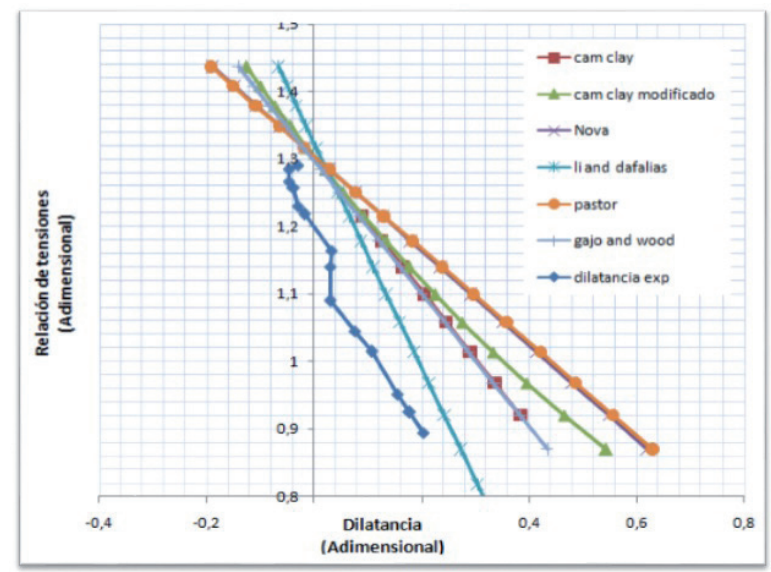

Figura 3. Modelamiento del fenómeno de dilatancia muestra Banding B13. 
En la Figura 3, se puede observar nuevamente la dilatancia medida (azul oscuro), en este caso los modelos se ajustan un poco más, gracias a la presión de confinamiento baja. Hay varios modelos que describen muy bien el comportamiento, pero el de Li and Defalias es el mejor (azul claro).

\section{ARENA BANDING B 14 (CASTRO 1969)}

Es una arena suelta también, con las mismas características que la anterior, a diferencia que acá se trabajara con una presión inicial de confinamiento de 98 Kpa. Ver Figura 4.

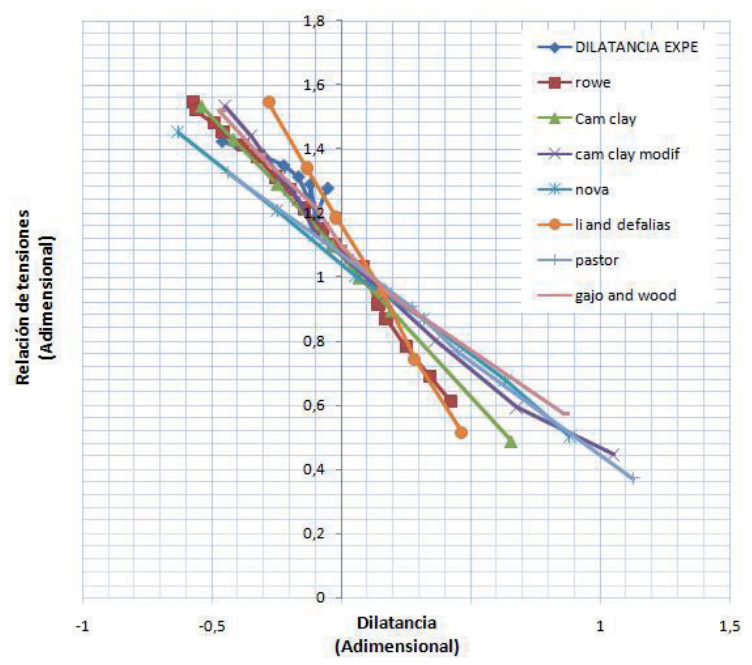

Figura 4. Modelamiento del fenómeno de dilatancia muestra Banding B14.

A pesar de que la dilatancia medida de la arena Banding B14 [26] es negativa (azul oscuro), los modelos logran predecir el comportamiento contractivo al momento de que se ejercen los esfuerzos en la arena.

Las ecuaciones alcanzan su predicción debido a que la presión de confinamiento inicial no es considerada grande, por otro lado, el mejor modelo que describe el fenómeno es el de Gajo and Wood.

\section{ARENA BANDING B43 (CASTRO 1969)}

Es el mismo tipo de arena, pero se trabajó con una presión de confinamiento más alta $(392 \mathrm{Kpa})$.

Se volvió a modelar el comportamiento con todos los parámetros necesarios para las ecuaciones y se obtuvo la siguiente grafica (Figura 5).

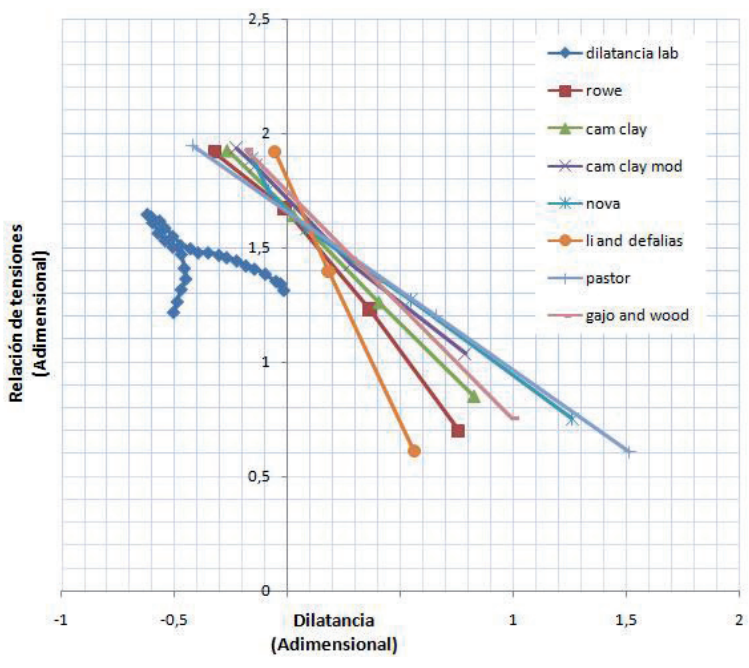

Figura 5. Modela miento del fenómeno de dilatancia muestra Banding B43.

Para arenas sueltas como es el caso de la arena Banding B43, la arena tiende a sufrir un proceso de contracción, contrario a la dilatancia. Al estar sometida a una presión de confinamiento inicial considerablemente grande, como se puede ver en la Figura 5 ningún modelo representa el comportamiento de la roca.

\section{RESULTADOS}

Al modelar el fenómeno de dilatancia, lo primero que se puede observar es que cuando la arena es suelta hay una dilatancia negativa o contracción, (arena B14 y B43) mientras que cuando la arena es densa existe la dilatancia positiva, o aumento de volumen (arena B13, 4479-E TCD50 y 4479-A TCD 300).

Otra característica es que cuando la presión inicial de confinamiento es alta, los modelos matemáticos no se ajustan, a medida que se aumenta la presión los ecuaciones se alejan de representar el fenómeno adecuadamente. Esto se puede observar en la arena B43 y 4479-A TCD 300.

El modelo que más se ajusto fue el de Li and Defalias, seguido por el de Gajo and Wood, cronológicamente son los últimos modelos propuestos, Li and Defalias tiene en cuenta la porosidad de la roca, por ésta razón puede que sea el más exacto.

\section{CONCLUSIONES}

- El fenómeno de dilatancia, va relacionado con el criterio de falla de la arena, y por ende con 
el problema del fenómeno de arenamiento y la estabilidad del pozo mientras se perfora. El correcto estudio de ésta propiedad puede mejorar la eficiencia en los procesos, pues se puede prevenir de mejor manera aquellos posibles problemas que van ligados con la inestabilidad geomecanica del pozo.

- Una formación con alto grado de dilatancia, es más susceptible a que se falle mientras se perfora o mientras se está produciendo hidrocarburo, a una con poca dilatancia.

- Todos los modelos matemáticos planteados para el cálculo de la dilatancia tienen en cuenta los esfuerzos sometidos en la roca.

- Un factor decisivo para que los modelos puedan ajustarse al comportamiento dilatante es la presión inicial de confinamiento de orden bajo, esto para que las partículas de arena logren acomodarse y puedan generar vacíos más grandes (mayor porosidad), por el contrario, entre mayor sea la presión de confinamiento, menor será la efectividad de las ecuaciones para modelar el fenómeno de dilatancia.

- El modelo que predice de mejor manera el comportamiento dilatante de las arenas es el propuesto por Li \& Dafalias. Este modelo es el que mostro un mejor ajuste con respeto a las mediciones experimentales, posiblemente porque dentro de su formulación posee diferentes parámetros en los cuales no solo interviene propiedades intrínsecas de la arena, si no propiedades como la relación de vacíos, la cual es crucial para determinar una propiedad fundamental como lo es la porosidad.

- La arena miga puede ser modelada en cuanto a la presión de confinamiento sea menor de $100 \mathrm{Kpa}$, y esto es general para todas las arenas en cuanto se quiera modelar el fenómeno de dilatancia. Cuando se tiene presiones de confinamiento mayores a estos valores, la dilatancia no puede llegar a modelarse

\section{REFERENCIAS}

1. Barden, L., Ismail, H. \& Tong, P. 1969. Plane strain deformation of granular material at low and high pressures. Gkotechnique 19, No. 4,441452.

2. Billam, J. 1972. Some aspects of the behaviour of granular materials at high pressures. In Stress- strain behaviour of soils. DD. 69-80 (ed. R. H. G. Parry).

3. BiTech Publishers Ltd. pp. 55-62. Ishihara, K. 1993. Thirty-third Rankine Lecture: Liquefaction and flow failure during earthquakes. Géotechnique, 43(3): 349-415.
4. Blümel, M. \& Bezat, F. A. 1998. Advanced control techniques for direct shear testing of jointed rock specimens.

5. Blümel, M., Button, E. A. \& Pötsch, M. 2002. Steifigkeits- abhängigies Scherverhalten von Fels. Felsbau Rock and Soil Engineering 20(3): 22-32.

6. Brosch, F. J., Schachner, K., Blümel, M., Fasching, A. \& Fritz, H. 2000. Preliminary investigation results on fabrics and related physical properties of an anisotropic gneiss. Journal of Structural Geology 22(11-12): 1773-1787.

7. Chen. Z. (s.f.). : "Analysis of a microcrack model and constitutive equations for time-dependent dilatancy of rocks". Geophysical Journal International.

8. Collins, I.F 2005: "Elastic/plastic models for soils and sands". Int. J. Mech. Sci. 47, 493-508

9. Cornforth, D. H. 1964. Some experiments on the influence of strain conditions on the strength of sand. Gkotechnique 14, No. 2, 143-167.

10. Dafalias, Y.F., Herrmann, L.R. 1986: "Bounding surface plasticity II: Application to isotropic cohesive soils". J. Eng. Mech. 112(12), 1263-1291.

11. De Josselin de Jong, G. 1976. Rowe's stressdilatancy relation based on friction. Géotechnique, 26(3): 527-534.

12. Diego Guillermo Manzanal, 2008:"Modelo constitutivo basado en la teoría de la plasticidad generalizada con la incorporación de parámetros de estado para arenas saturadas y no saturadas". Universidad politécnica de Madrid.

13. Dong, J.-J. \& Pan, Y.-W. 1996.A Hierarchical Model of Rough Rock Joints Based on Micromechanics. Int. J. Rock Mech. Min. Sci. Geomech. Abstr. 33(2): 111-123.

14. Foulis. Bishop, A. W. 1972. Shear strength parameters for undisturbed and remoulded soils specimens. In Stress-strain behaviour of soils, pp. 3-58 (ed. R. H. G. Parry).

15. Foulis. Bishop. A. W. \& Green, G. E. 1965. The influence of end restraint on the compression strength of a cohe- sionless soil. GPotechniaue 15, No. 3,243-266. 
16. Gao, Z.W., Zhao, J.D. 2012: “Constitutive modeling of artificially cemented sand by considering fabric anisotropy". Computer and Geotechnics 41, 57-69.

17. Gajo, A. \& Muir Wood, D. 1999. : “A kinematic hardening constitutive model for sands: the multiaxial formulation". Int. J. Numer. Anal. Methods Geomech. 23, No. 5, 925-965.

18. Gentier, S., Riss, J. Archambault, G., Flamand, R. $\&$ Hopkins, D. 2000. Influence of fracture geometry on shear behaviour. Int. J. Rock Mech. Min. Sci. 37: 161-174.

19. Grasselli, G., Wirth, J. \& Egger, P. 2002. Quantitative three- dimensional description of a rough surface and parameter evo- lution with shearing. Int. J. Rock Mech. Min. Sci. 39, 789-800.

20. Grupo geotecnia. (s.f.). : "Deformabilidad y resistencia de los suelos". Universidad de Cantabria.

21. Hughes, J. M. O., Wroth, C. P. \& Windle, D. 1977. Pressuremeter tests in sands. Ghotechnique 27, No. 4, 455477.

22. Horsfield, D., and Been, K. 1987. Computer controlled triaxial testing. In Proceedings of the 1st Canadian Symposium on Microcomputer Applications to Geotechnique, Regina, Sask. 22-23 October 1987.

23. Indraratna, B. \& Haque, A. 2000. "Shear Behaviour of Rock Joints. Balkema: Rotterdam, Brookfield.

24. In Marr \& Fairhurst (eds.), Nondestructive and Automated Testing for Soil and Rock Properties. ASTM STP 1350, Am. Soc. Test. Mat., 276-289.

25. Jefferies, M.G., and Been, K. 2000. Implications for critical state theory from isotropic compression of sand. Géotechnique, 50(4): 419-429.

26. Jefferies, M.G., and Shuttle, D. 2002. Dilation in general Cam- bridge-type models. Géotechnique, 52(9): 625-638.

27. Jefferies, M.G. 1997. Plastic work and isotropic softening in un- loading. Géotechnique, 47(5): 1037-1042.

28. Ken Been and Michael Jefferies, 2004": Stressdilatancy in very loose sand" NRC Research Press.
29. Koerner, R. M. 1970. Behaviour of single mineral soils in triaxial shear. J. Soil Mech. Fdns Div. Am. Sot. Civ. Engrs 96, SM4, 1373-1390.

30. Ladanyi, B. \& Archambault, G. 1970. "Simulation of shear behavior of a jointed rock mass". In Proc. 11th Symp. on Rock Mechanics: Theory and Practice, AIME, 105-125: NewYork.

31. Ladanyi, B. 1960. Etude des relations entre les contraintes et des $\mathrm{d} \&$ formations lors du cisaillement des sols pulvtrulents. Annls Trau. Publ. Belg. No. 3, 1-30.

32. Li, X.-S., Dafalias, Y. F., and Wang, Z.-L. 1999:"State-dependent dilatancy in critical-state constitutive modeling of sand." Can. Geotech. J. (3) 64, 599-611.

33. Marachi, N. D., Chan, C. K., Seed, H. B. \& Duncan, J. M. 1969.: "Strength and deformation characteristics of rockJill materials". University of California Report TE-69-5, Berkeley.

34. Mateos García 1995. : "Determinación experimental de la función de dilatancia en un suelo granular denso". J. Geogaceta (18) 150-152.

35. Nova, R., and Wood, D. M. 1979. : "A constitutive model for sandin triaxial compression." Int. J. Numer. Analyt. Meth. Geomech., 255-278.

36. Pastor, M., and Zienkiewicz, O. C. 1986.: "A generalized plasticity, hierarchical model for sand under monotonic and cyclic loading."

37. Proc., 2nd Int. Symp. on Numerical Models in Geomechanics, 131-150.

38. Pestana, J.M., Whittle, A.J., Gens, A. 2002.: "Evaluation of a constitutive model for clays and sands: Part II - clay behaviour". Int. J. Numer. Anal. Meth. Geomech. 26, 1123-1146.

39. Reynolds, O. 1885: "On the dilatancy of media composed of rigid particles in contact. With experimental illustrations". Phil. Mag. 20, 469-482

40. Roscoe \& Burland 1968. : "On the generalized strain-strain behaviour of wet clay". Engineering plasticity, J. Heyman and F.A.Cambridge University press, Cambridge UK 535-609 
41. Rowe, P. W. 1962. : "The stress-dilatancy relation for static equilibrium of an assembly of particles in contact", Proc. Roy. Soc., London, A269, 500-527.

42. Seywald, C. 2006. Investigations on the relationship between surface roughness and dilation of rock joints in direct shear. DiplomaThe- sis, Institute for Rock Mechanics andTunnelling, Graz University of Technology, Austria.

43. Schieg, T. 2006. Investigations on the shear behaviour of artificial rock joints. Diploma Thesis, Institute for Rock Mechanics and Tunnelling, Graz University of Technology, Austria.

44. Schofield, A.N. 1998: "The Mohr-Coulomb error. In: Proc. Symp. on Mechanics and Geotechnics", LMS Ecole Polytechnique, Paris, vol. 23, pp. 19-27

45. Sridharan, A. and Prakash, K. 1995: Discussion on 'Limitations of Conventional Analysis of Consolidation Settlement', ASCE, J. of Geotechnical
Engineering, 121 (6): 517.

46. Taylor, D.W. 1948. : "Fundamentals of soil mechanics", john wiley and sons, Inc. New York.

47. Vaid, Y.P., and Sasitharan, S. 1992. The strength and dilatancy of sand. Canadian Geotechnical Journal, 29: 522-526.

48. Wood, D.M. 1990. Soil behaviour and critical state soil mechanics. Cambridge University Press, London, UK.

49. Wroth y Schofield 1963. : Ed. Mcgraw-Hill publishing Company Ltd.

50. Yamamuro, J.A., and Lade, P.V. 1997. Static liquefaction of very loose sands. Canadian Geotechnical Journal, 34: 905-917.

51. Yang, J. 2002. Non-uniqueness of flow liquefaction line for loose sand. Géotechnique, 52(10): 757-760.

Recepción: 28 de Agosto de 2015

Aceptación: 27 de Octubre de 2015 


\section{ANEXOS}

Parámetros para los diferentes tipos de arena.

\section{Anexo A.}

\begin{tabular}{|c|c|}
\hline \multicolumn{2}{|r|}{ ARENA DE MIGA } \\
\hline$\lambda$ & 0,0308 Pendiente de LEC en el plano e-p' \\
\hline$£$ & 0,6 parámetro de ajuste de LEC \\
\hline $\begin{array}{l}\text { h1 } \\
\text { h2 }\end{array}$ & $\begin{array}{l}\text { Constante de variación } M_{f} \text { con el parámetro de estado } \\
1,02 / 0,15\end{array}$ \\
\hline do & 0,65 Multiplicador de dilatancia \\
\hline m & 2,52 Parámetro asociado a la linea de transformación \\
\hline H'o & 80 Parámetro de endurecimiento plástico isótropo \\
\hline$\beta^{\prime} 0$ & $\begin{array}{l}\text { 1,1 Parámetro al m. plástico isótropo y variación con parámetro de } \\
\text { estado }\end{array}$ \\
\hline $\mathrm{H}_{\mathrm{vo}}$ & 25 Constante del módulo plástico volumétrico \\
\hline$\beta v$ & 3,98 Parámetro asociado a la resistencia de pico \\
\hline
\end{tabular}

\section{ANEXo B}

\begin{tabular}{|c|c|}
\hline \multicolumn{2}{|r|}{ ARENA DE TOYOURA } \\
\hline$\lambda$ & 0,019 Pendiente de LEC en el plano e-p' \\
\hline$£$ & parámetro de ajuste de LEC \\
\hline h1,h2 & 1,31/0,85 Constante de variación $\mathrm{M}_{\mathrm{f}}$ con el parámetro de estado \\
\hline do & 0,88 Multiplicador de dilatancia \\
\hline m & 3,5 Parámetro asociado a la línea de transformación \\
\hline H'o & 125 Parámetro de endurecimiento plástico isótropo \\
\hline & $\begin{array}{l}\text { 1,9 Parámetro al m. plástico isótropo y variación con parámetro de } \\
\text { estado }\end{array}$ \\
\hline Hvo & 175 Constante del módulo plástico volumétrico \\
\hline & 1,5 Parámetro asociado a la resistencia de pico \\
\hline
\end{tabular}

\section{ANEXO C}

\begin{tabular}{|c|c|c|}
\hline \multicolumn{3}{|r|}{ BANDING O "B" } \\
\hline$\lambda$ & 0,0136 & Pendiente de LEC en el plano e-p' \\
\hline$£$ & 0,6 & parámetro de ajuste de LEC \\
\hline h1 h2 & $1,17 / 0,81$ & Constante de variación $\mathrm{M}_{\mathrm{f}}$ con el parámetro de estado \\
\hline do & 0,73 & Multiplicador de dilatancia \\
\hline m & 2,02 & Parámetro asociado a la línea de transformación \\
\hline H'o & 125 & Parámetro de endurecimiento plástico isótropo \\
\hline$\beta^{\prime} 0$ & & $\begin{array}{l}\text { Parámetro al m. plástico isótropo y variación con parámetro de } \\
\text { estado }\end{array}$ \\
\hline Hvo & 165 & Constante del módulo plástico volumétrico \\
\hline$\beta v$ & 1,82 & Parámetro asociado a la resistencia de pico \\
\hline
\end{tabular}

\section{ANEXO D}

\begin{tabular}{|c|c|}
\hline \multicolumn{2}{|r|}{ Arena Speswhite } \\
\hline$\lambda$ & 0,11 Pendiente de LEC en el plano e-p' \\
\hline$£$ & parámetro de ajuste de LEC \\
\hline h1 h2 & 1/0,4 Constante de variación $\mathrm{M}_{\mathrm{f}}$ con el parámetro de estado \\
\hline do & 0,98 Multiplicador de dilatancia \\
\hline m & 5,6 Parámetro asociado a la línea de transformación \\
\hline H'o & 25 Parámetro de endurecimiento plástico isótropo \\
\hline$\beta^{\prime} 0$ & $\begin{array}{l}\text { 0,5 Parámetro al m. plástico isótropo y variación con parámetro de } \\
\text { estado }\end{array}$ \\
\hline Hvo & 6 Constante del módulo plástico volumétrico \\
\hline$\beta v$ & 0,5 Parámetro asociado a la resistencia de pico \\
\hline
\end{tabular}

\section{ANEXo E}

\section{ARENA KURNELL}

\begin{tabular}{|c|c|c|}
\hline$\lambda$ & 0,0328 & Pendiente de LEC en el plano e-p' \\
\hline$\varepsilon$ & 0,6 & parámetro de ajuste de LEC \\
\hline h1 h2 & $1 / 0,55$ & Constante de variación $\mathrm{M}_{\mathrm{i}}$ con el parámetro de estado \\
\hline do & 0,8 & Multiplicador de dilatancia \\
\hline m & 3,32 & Parámetro asociado a la linea de transformación \\
\hline $\mathrm{H}^{\prime} \mathrm{O}$ & 135 & Parámetro de endurecimiento plástico isótropo \\
\hline$\beta^{\prime} 0$ & 1,1 & $\begin{array}{l}\text { Parámetro al m. plástico isótropo y variación con parámetro de } \\
\text { estado }\end{array}$ \\
\hline Hvo & 20 & Constante del módulo plástico volumétrico \\
\hline$\beta v$ & 0,95 & Parámetro asociado a la resistencia de pico \\
\hline
\end{tabular}

